\title{
Cryoglobulins are not essential
}

In 1933 Wintrope and Buell described "an extraordinary hyperproteinemia" in a patient suffering from multiple myeloma. She "presented symptoms of coldness, blanching and a peculiar mottling of the extremities, as well as other signs of disturbed circulation, and was found to have, in her blood, a voluminous quantity of a substance which invariably was precipitated immediately on withdrawal of the blood from the body".

Since then several authors have analysed the cryoprecipitation reaction as well as the cryoprecipitated proteins. ${ }^{2}{ }^{3}$ In 1974, Brouet and colleagues proposed a classification based on the type of protein that constitutes the cryoprecipitate. ${ }^{4}$ This classification has always had the disadvantage of associating diseases with very different aetiology and outcome, in the same group. In addition to cryoglobulinaemia secondary to well defined pathologies, Brouet and colleagues realised that a large proportion $(30 \%)$ of patients had no known disease. This group of idiopathic cryoglobulinaemia has been referred to as "essential" cryoglobulinaemia. Since our initial report in $1990,{ }^{5}$ it has become clear that most of these essential cryoglobulinaemias are in fact associated with hepatitis $C$ virus infection. The description of a cryoglobulinaemia therefore has to take into account this important new finding.

\section{What is a cryoglobulin?}

The definition is based on an in vitro observation. A cryoglobulin is a globulin that precipitates when the serum is incubated at a temperature $<37^{\circ} \mathrm{C}$. Each cryoglobulin has an individual thermal curve with well defined kinetics of precipitation. Sometimes there is a good correlation between the thermal amplitude and the clinical signs (purpura after cold exposure) but in general cryoprecipitation is perceived as an in vitro artefact, with little or no direct pathological consequences in organs other than the skin. Indeed it is thought that the accumulation of cryoglobulins, for instance in the renal glomeruli, could not be explained for by a decrease in temperature. In a model of cryoglobulinaemia it could be demonstrated that when mice were kept in an external environment of $37^{\circ} \mathrm{C}$ no disease developed, whereas skin vasculitis and glomerulonephritis were the hallmarks of mice kept at normal room temperature $\left(20^{\circ} \mathrm{C}\right) .^{6}$ Probably cryoimmunecomplexes developed under low temperature conditions in the skin, were dragged off by the blood stream and deposited in the kidney before having been redissolved.

\section{Classification of cryoglobulins}

The classification proposed by Brouet and colleagues ${ }^{4}$ in 1974 is clearly the most used and the best accepted.

Type I includes cryoglobulins consisting of a monoclonal immunoglobulin (Ig) only (paraprotein), most frequently IgM, occasionally IgG, and rarely IgA. These cryoglobulins are mostly found in patients with lymphoproliferative diseases including multiple myeloma, Waldenström's macroglobulinaemia, non-Hodgkin's lymphoma, and chronic lymphocytic leukaemia. Some cases represent monoclonal gammopathia of undetermined significance.

Type II includes cryoglobulins consisting of a mixture of immunoglobulins of different isotype, one of the immunoglobulins being a monoclonal Ig. The monoclonal Ig is an antibody directed at the Fc portion of normal IgG-that is, it is a rheumatoid factor (RF). The association between the monoclonal RF and polyclonal IgG produces immune complexes that precipitate in the cold. In general the monoclonal $\mathrm{RF}$ is an $\operatorname{IgM}$, however, IgG and IgA have been reported. The most frequent causes of this type of cryoglobulinaemia are chronic hepatitis $\mathrm{C}$ virus (HCV) infection, macroglobulinaemia, and Sjögren's syndrome. ${ }^{78}$

Type III cryoglobulins are constituted by a mixture of polyclonal immunoglobulins belonging to different isotypes. In practice the association of IgM and IgG polyclonal antibodies is the most frequent combination. Very often the IgM contains polyclonal RF, which react with the IgG. Such cryoglobulins are observed during many viral (including $\mathrm{HCV}$ ), bacterial or parasitic infections, as well as in a series of autoimmune and tumoral disorders. Analysis of cryoglobulins type III from patients with chronic HCV by immunoblotting ${ }^{9}$ or by bidimensional electrophoresis ${ }^{10}$ has shown that many contain oligoclonal rather than polyclonal IgM. This type of cryoglobulin could represent an intermediate state, and maybe an evolution from type III to type II cryoglobulins - that is, from a state of polyclonal IgM RF production towards a state of monoclonality.

Many authors have used other terms such as "mixed cryoglobulins" in contrast with monoclonal cryoglobulins. Mixed cryoglobulins correspond to type II and type III of Brouet. Finally, the existence of cryoglobulins that do not fit well into any of the categories described above should be noted.

\section{Cryoglobulin measurements today}

The methods used for the quantification of cryoglobulins are not uniform. No international committee has tried to standardise cryoglobulin measurements. Therefore cryoglobulins are either frequently or rarely observed in a particular disease depending on the definition used and the way in which the cryoglobulin was quantified. Some aspects of the measurements are however respected by everybody. Firstly, the blood collected must be maintained at $37^{\circ} \mathrm{C}$ from the start so as not to lose cryoglobulins, which precipitate as soon as the temperature falls below $37^{\circ} \mathrm{C}$. Once the blood has coagulated the serum is separated by centrifugation at $37^{\circ} \mathrm{C}$. The serum should then be freed of fibrinogen, which could, like several other plasma proteins, precipitate in the cold. It is clear that this separation cannot be perfect in patients who are anticoagulated, particularly when receiving intravenous heparin. The serum is left for four days between $0^{\circ} \mathrm{C}$ and $4^{\circ} \mathrm{C}$. The serum is then centrifuged and the cryocrit measured in a similar way as a haematocrit. The cryocrit will of course depend on the speed and length of centrifugation, both of which have not been standardised. In this test, the definition of a cryoglobulin will also depend on its "visibility". A small amount of cryoglobulins will not be visible and a cryoglobulin with a special physical aspectlike a cryogel - can be missed. An alternative is to measure the quantity of proteins that remain attached to the bottom of the tube after centrifugation. Here there are also differences from one laboratory to another. Should the precipitate be washed, how often, and in which buffer? Should it be resuspended at $37^{\circ} \mathrm{C}$ and only measure the solubilised proteins? From our experience these parameters can completely modify the results. Each wash is associated with a loss of protein resulting from dilution and maybe also result from a modification of the medium (for example, loss of factors that favour precipitation in the cold such as fibronectin). In addition a large fraction of cryoproteins do not redissolve, or only partially redissolve. 
Lastly, the conditions that are optimal for one cryoglobulin are not necessarily optimal for another one because of variability of cryoglobulins physical and chemical properties. In many laboratories a cryoglobulin is defined by the presence of more than $50 \mu \mathrm{g}$ of protein per millilitre of serum after 96 hours of incubation at $4^{\circ} \mathrm{C}$ and three washes with a phosphate- $\mathrm{NaCl}$ buffer at $4^{\circ} \mathrm{C}$. Also, different techniques like immunoelectrophoresis, immunofixation, immunoblotting or bidimensional electrophoresis make it possible to determine the isotype and clonality of cryoglobulins in the cryoprecipitate, and exclude the presence of another cryoprotein (cryoalbumin, cryofibrinogen, etc).

\section{What are the most frequent clinical signs?}

In many patients the clinical presentation includes the signs of the underlying disease. Only those thought to be specific for cryoglobulins will be listed here.

Cryoglobulinaemia leads to systemic vasculitis because of a direct obstruction of the vessels, or to inflammation induced by the deposition of the Ig aggregate mediated to a large extent through complement activation. Most frequently small arteries and veins are involved. Purpura, with sometimes severe ulcerations of the legs, is described in almost $100 \%$ of the patients. Other skin manifestations include urticaria, Raynaud's phenomenon with sometimes acral necrosis. Clinical relevant distal symmetric sensomotorical polyneuritis is frequent (in $20 \%$ of patients and if systematically explored up to $86 \%{ }^{11}$ ), whereas the more dramatic acute mononeuritis (multiplex) or stroke are seen more rarely. Abdominal pain $(20 \%)$ is best explained by vasculitis of the gastrointestinal tract. Membranoproliferative glomerulonephritis (MPGN) is found in many patients, although the prevalence is difficult to estimate. Probably MPGN occurs in around $10-30 \%$ of patients often as a late and severe manifestation of the disease. $^{2} 47812-14$ It is accompanied by hypertension. The arthralgia is a frequent symptom $(70 \%)$, although it rarely evolves into real arthritis. No organ is spared from the possible damage of cryoglobulins and the clinician should include cryoglobulinaemia in its differential diagnosis when confronted with any form of systemic vasculitis. ${ }^{14}$

\section{Prognosis and treatment}

Most often the underlying disease process determines the prognosis. For example in type I cryoglobulinaemia, the prognosis is certainly given by the proliferation of the $\mathrm{B}$ cell clone-that is, multiple myeloma versus monoclonal gammopathia of underdetermined significance. Liver failure occurs in patients with cryoglobulinaemia secondary to HCV hepatitis. Lymphoma complicates many cases of cryoglobulinaemia secondary to Sjögren's syndrome. However, in many patients $(30 \%)$, the vasculitis is responsible for irreversible lesions and death. Corticosteroids have been used in an attempt to block the vasculitic process and reported to produce short-term benefit, but whether they improve the overall survival is not known. Immunosuppression has been used in patients with cryoglobulinaemia secondary to autoimmune diseases with some success. It is ironic to see that "mixed essential cryoglobulinaemia" had been considered to be of autoimmune origin and treated as such for years-inducing partial remissions according to many authors. The specific treatment of $\mathrm{HCV}$ infection in these patients with interferon over the past years has however produced much better results. Much attention has been devoted to remove the damaging cryoglobulins particularly in patients having a monoclonal component (type I and II). Intensive plasmapheresis reduces purpura and skin lesions and improves the healing of skin ulcers. Occasionally for the patients in whom the very high amount of cryogobulins was responsible for vascular obstruction, the effect might even be excellent (for example, reversal of anuric renal failure caused by plugging of the glomeruli). It has however little effect on already established tissue destruction. Plasmapheresis is costly and cumbersome for many patients, who would prefer their leg ulcers rather than attending regular sessions of plasma removal. Particular attention should also be given to the replacement volume. When only sodium choloride is given, oedema might develop because of hypoalbuminaemia. Fresh frozen plasma has too many drawbacks to be used (anaphylactic reactions, transmission of infections, etc) and IgG replacement for preventing hypoIgG is dangerous in those patients who have a monoclonal (or polyclonal) RF. Indeed, adding the Ag (IgG) in a disease state characterised by large amounts of $\mathrm{Ab}(\mathrm{RF})$ may induce acute immune complex disease.$^{15}$ Finally, some patients have signs of anaphylaxis when the procedure is not carried out at $37^{\circ} \mathrm{C}$.

\section{The special case of $\mathrm{HCV}$ associated cryoglobulinaemia}

The high prevalence of liver disease in patients with mixed essential cryoglobulinaemia, as reported until 1990, is in retrospect very easy to understand because cryoglobulins (type II and III) are a hallmark of chronic HCV infection.

A high prevalence of mixed cryoglobulins in chronic $\mathrm{HCV}$ infection has been reported in many studies. ${ }^{8}{ }^{16}$ In the study by Lunel et al, ${ }^{17}$ mixed cryoglobulins were detected in five of 136 normal blood donors (concentrations between 0.02 and $0.03 \mathrm{~g} / \mathrm{l})$. About one third of patients with chronic liver diseases (alcoholic, viral but non-A non-B non-C, autoimmune and primary biliary cirrhosis) had low concentrations of cryoglobulins (mean (SD)) (0.08 (0.03) $\mathrm{g} / \mathrm{l})$, most of these had advanced disease with significant hypergammaglobulinaemia, hypoalbuminaemia, and cirrhosis. Only six of 40 patients (15\%) with chronic hepatitis $\mathrm{B}$ had detectable cryoglobulins - that is, a prevalence that did not differ from other liver diseases. The six positive patients had also higher gammaglobulin values than the other patients with chronic hepatitis B. Thus the association of cryoglobulinaemia and hepatitis B is certainly not specific and the disease entity cryoglobulinaemia/hepatitis $\mathrm{B}$ should be questioned. ${ }^{18}{ }^{19}$ The association between cryoglobulins and chronic hepatitis C infection was evident: 69 of 127 (54\%) patients were positive (mean value $=0.23 \mathrm{~g} / \mathrm{l}$, range $0.03-1.5 \mathrm{~g} / \mathrm{l}$ ), 22 had a cryoglobulin type II and 47 had a cryoglobulin type III. Only 18 of 69 patients had clinical signs of generalised vasculitis. The cryoprecipitates were rich in HCV RNA and in HCV antibodies suggesting the formation of specific immune complexes, although coprecipitation in vitro is certainly not sufficient evidence for the existence of circulating immune complexes. ${ }^{17} 2021$ Liver cirrhosis and a longer duration of clinical or biological signs of hepatitis were both significantly associated with the presence of cryoglobulins confirming previous analyses. ${ }^{16}$

The finding of RF is common in chronic $\mathrm{HCV}$ infection $(75-100 \%) .^{22-24}$ The formation of $\mathrm{RF}$ (anti-IgG)/IgG immune complexes is the major step preceding cryoprecipitation. RFs are found in many chronic infectious diseases other than HCV infection and their presence does not necessarily mean autoimmunity. In rheumatoid arthritis, the IgM RF that has been cloned show often multiple somatic mutations, which would indicate a specific immune response against a specific antigen. On the other hand, monoclonal IgM from patients with chronic lymphocytic leukaemia shows only exceptionally such mutations indicating a germ-line configuration. Recently Crouzier et al analysed the monoclonal RF 
Table 1 Prevalence of HCV infection in patients with suspected cryoglobulinaemia

\begin{tabular}{lrlll}
\hline & \multicolumn{4}{l}{$H C V$ serology } \\
\cline { 3 - 5 } Cryoglobulins $(\mathrm{mg} / \mathrm{ml})$ & Patients & Positive & Negative & Positive (\%) \\
\hline$\leqslant 0.05 \dagger$ & 133 & 0 & 13 & 0 \\
$>0.05$ and $\leqslant 0.25$ & 82 & 3 & 25 & 11 \\
$>0.25$ and $\leqslant 1.00$ & 18 & 5 & 6 & 45 \\
$>1.00$ & 7 & 4 & 1 & 80 \\
\hline
\end{tabular}

${ }^{\star} \mathrm{HCV}$ serology was performed in only a fraction of the patients. $\dagger \leqslant 0.05 \mathrm{mg} / \mathrm{ml}$ is reported in our laboratory as negative for cryoglobulins.

producing B lymphocytes from a patient with chronic $\mathrm{HCV}$ infection ${ }^{25}$ and found clear evidence for some degree of somatic mutations. This preliminary finding would suggest that the RF in HCV infection is something different from a true "malignant" clonal proliferation but is also not identical to the RF of autoimmune diseases.

In conclusion, cryoglobulins are often found in patients with HCV infection and a necessary step for their development seems to be long standing infection with liver disease and hypergammaglobulinaemia. Because chronic $\mathrm{HCV}$ infection often progresses without any clinical or laboratory abnormality, only a liver biopsy will show the presence of hepatitis in many patients. ${ }^{26}$ The absence of hepatitis on liver biopsy in a patient with cryoglobulinaemia and HCV infection (tested by the presence of $\mathrm{HCV}$ RNA and a positive serology) is rare enough to merit publication. ${ }^{27}$ However, it may be possible that chronic liver disease is not an obligatory step in the progression towards cryoglobulinaemia. The $\mathrm{HCV}$ is also found in different types of leucocytes ${ }^{28}$ and may stimulate the production of cryoglobulin by itself or with the help of another factor.

Finally, it is worth emphasising that the prevalence of $\mathrm{HCV}$ infection in patients with suspected cryoglobulinaemia is high. In our laboratory the prevalence increased directly with the amount of cryoglobulins detected (see table 1), indicating that $\mathrm{HCV}$ has to be looked for in patients with high values of cryoglobulins.

\section{Sjögren's syndrome and lymphoma}

Cryoglobulins are often found in patients with Sjögren's syndrome $\mathrm{e}^{29}$ and the frequent progression of this disease to malignant lymphoma has been recognised by many authors (probably around 30\%). A sicca syndrome is frequently found in HCV cryoglobulinaemia, however the histology of the salivary glands and more so the presence of specific $\mathrm{Ab}$ (Ro/SS-A) for Sjögren's syndrome permits a clear distinction between the two. ${ }^{30} 31$ Although HCV cryoglobulinaemia can progress to lymphoma, it is most often of low grade and exceptionally is responsible for the death of the patient.

\section{Conclusion}

Cryoglobulins have fascinated clinicians for 65 years. Today most patients having clinical signs of cryoglobulinaemia have a well identifiable disease. The most frequent cause is chronic $\mathrm{HCV}$ infection with liver damage. Interferon induces remission in half the patients. A B cell line clone, in case of monoclonal cryoglobulinaemia, can be treated appropriately with chemotherapy and possibly in the future with intensive chemotherapy and the support of peripheral stem cells. Sjögren's syndrome should be recognised as well, so as to provide the appropriate treatment. The same is true for chronic infections and leukaemia. Once these possibilities have been excluded, a small number of patients will remain with true idiopathic (essential) cryoglobulinaemia.
The personal work presented has been performed with the help of the Wissenschaftlicher Fonds des Departementes Innere Medizin am Kantonsspital Basel, and the Swiss National Science Foundation.

M TRENDELENBURG J A SCHIFFERLI

Immunonephrology Laboratory of the Medizinische Universitätsklinik B, Petersgraben 4, Kantonsspital, Basel, Switzerland

1 Wintrobe $M$, Buell M. Hyperproteinemia associated with multiple myeloma. With report of a case in which an extraordinary hyperproteinemia was With report of a case in which an extraordinary hyperproteinemia was
associated with thrombosis of the retinal veins and symptoms suggesting Raynaud's disease. Bull Johns Hopkins Hosp 1933;52:156-65.

2 Meltzer M, Franklin EC, Elias K, McCluskey RT, Cooper N. Cryoglobulinemia-A clinical and laboratory study. Am J Med 1966;40:837-56. 3 Wang AC. Molecular basis for cryoprecipitation. Springer Semin Immunopathol 1988;10:21-34.

4 Brouet JC, Clauvel JP, Danon F, Klein M, Seligmann M. Biologic and clinical significance of cryoglobulins. A report of 86 cases. Am J Med 1974;57: 775-88.

5 Pascual M, Perrin L, Giostra E, Schifferli JA. Hepatitis C virus in patients with cryoglobulinaemia type II. J Infect Dis 1990;162:569-70.

6 Fulpius T, Berney T, Lemoine R, et al. Glomerulopathy induced by IgG3 anti-trinitrophenyl monoclonal cryoglobulins derived from nonautoimmune mice. Kidney Int 1994;45:962-71.

7 Invernizzi F, Monti G, Zanussi C. The clinical spectrum of mixed cryoglobulinemia, Immunological aspects of malignant lymphomas and cryoglobulinemia. Milan: Edi-ermes, 1990: 221-33.

8 Ferri C, La Civita L, Longombardo G, Greco F, Bombardieri S. Hepatitis C virus and mixed cryoglobulinemia. Eur J Clin Invest 1993;23:399-405.

9 Musset L, Diemert MC, Taibi F, et al. Characterization of cryoglobulins by immunoblotting. Clin Chem 1992;38:798-802.

10 Tissot JD, Schifferli JA, Hochstraser DF, Pasquali C, Spertini F, Clement F, et al. Two-dimentional polyacrylamide gel electrophoresis analysis of cryoglobulins and identification of an IgM-associated peptide. J Immunol Methods 1994;173:63-75.

11 Ciompi ML, Marini D, Siciliano G, et al. Cryoglobulinemic peripheral neuropathy: neurophysiologic evaluation in twenty-two patients. Biomed Pharmacother 1996;50:329-36.

12 Misiani R, Bellavita P, Fenili D, et al. Hepatitis C Virus infection in patients with essential mixed cryoglobulinemia. Ann Intern Med 1992;117:573-7.

13 Willems M, Sheng L, Roskams T, et al. Hepatitis C Virus and its genotypes in patients suffering from chronic Hepatitis C with or without a cryoglobulinemia-related syndrome. J Med Virol 1994;44:266-71.

14 Gorevic P, Kassab HJ, Levo Y, et al. Mixed cryoglobulinaemia: Clinical aspects and long-term follow-up of 40 patients. Am J Med 1980;69:287-308

15 Barton J, Herrera G, Galla J, et al. Acute cryoglobulinemic renal failure after intravenous infusion of gamma globulin. Am J Med 1987;82:624-9

16 Schifferli JA, French LA, Tissot JD. Hepatitis C virus infection, cryoglobulinaemia and glomerulonephritis. In: Grunfeld JP, Bach JF, Kreis $\mathrm{H}$, naemia and glomerulonephritis. In: Grunfeld JP, Bach JF, Kreis H,

17 Lunel F, Musset L, Cacoub P, Frangeul L, Cresta P, Perrin M, et al. Cryoglobulinaemia in chronic liver diseases: role of hepatitis $\mathrm{C}$ virus and liver damage. Gastroenterology 1994;106:1291-300.

18 Johnson RJ, Couser WG. Hepatitis B infection and renal disease: clinical, immunopathogenic and therapeutic considerations. Kidney Int 1990;37: 663-76.

19 Monti G, Galli M, Invernizzi F, et al. Cryoglobulinemias: A multi-canter study of the early clinical and laboratory manifestations of primary and secondary disease. Q J Med 1995;88:115-26.

20 Bichard P, Ounanian A, Girard M, et al. High prevalence of hepatitis C virus RNA in the supernatant and the cryoprecipitate of patients with essential secondary type II mixed cryoglobulinaemia. J Hepatol 1994;21:58-63.

21 Agnello V, Chung RT, Kaplan LM. A role for hepatitis C virus infection in type II cryoglobulinaemia. N Engl J Med 1992;327:1490-5.

22 Clifford BD, Donahue D, Smith L, Cable E, Luttig B, Manns M, et al. High prevalence of serological markers of autoimmunity in patients with chronic hepatitis C. Hepatology 1995;21:613-19.

23 Levey JM, Bjornsson B, Banner B, Kuhns M, Malhotra R, Whitman N, et al. Mixed cryoglobulinaemia in chronic hepatitis $\mathrm{C}$ infection. A clinicopathologic analysis of 10 cases and review of recent literature. Medicine logic analysis of
$1994 ; 73: 53-67$.

24 Pawlotsky JM, Ben Yahia M, Andre C, Voisin MC, Intrator L, RoudotThoraval $\mathrm{F}$, et al. Immunological disorders in $\mathrm{C}$ virus chronic active hepatitis: a prospective case-control study. Hepatology 1994;19:841-8.

25 Crouzier R, Martin T, Pasquali JL. Monoclonal IgM rheumatoid factor secreted by CDs-negative $B$ cells during mixed cryoglobulinaemia. Evidence for somatic mutations and intraclonal diversity of the expressed VH region gene. J Immunol 1995;154:413-21.

26 Pechere-Bertschi A, Perrin L, de Saussure P, Widmann JJ, Giostra E, Schifferli JA. Hepatitis C: a possible etiology for cryoglobulinaemia type II. Clin Exp Immunol 1992;89:419-22.

27 Sechi LA, Pirisi M, Bartoli E. Membranoproliferative glomerulonephritis associated with hepatitis $\mathrm{C}$ infection with no evidence of liver disease. JAMA 1994;271:194.

28 Pozzato G, Mazzaro C, Crovatto M, Modolo ML, Ceselli S, Mazzi G, et al. Low-grade malignant lymphoma, hepatitis $C$ virus infection, and mixed cryoglobulinaemia. Blood 1994;84:3047-53.

29 Tzioufas AG, Manoussakis MN, Costello R, et al. Cryoglobulinemia in autoimmune rheumatic diseases. Arthritis Rheum 1986;29:1098-104.

30 Tzioufas A, Boumba D, Skopouli F, Moutsopoulos H. Mixed monoclonal cryoglobulinemia and monoclonal rheumatoid factor cross-reactive idiotypes as predictive factors for the development of lymphoma in primary otypes as predictive factors for the development of lym
Sjögren's syndrome. Arthritis Rheum 1996;39:767-72.

31 Jorgensen C, Legouffe MC, Perney P, et al. Sicca syndrome associated with hepatitis C virus infection. Arthritis Rheum 1996;39:1166-71. 